\title{
Detection of gas reservoirs by the joint use of P- and PS-waves: A case study on the Ordos basin, China*
}

\author{
Xiucheng Wei ${ }^{1, \star}$ Xiangyang $\mathrm{Li}^{2} \quad$ Yang $\mathrm{Liu}^{3}$ \\ Songqun $\mathrm{Shi}^{4}$ and Weidong Jiang ${ }^{5}$ \\ ${ }^{1}$ Petroleum Exploration and Production Institute, China Petroleum and Chemical Corporation, \\ Beijing 100083, China \\ ${ }^{2}$ Edinburgh Anisotropy Project, British Geological Survey, Edinburgh EH9 3LA, UK \\ ${ }^{3}$ Faculty of Resources and Information, China University of Petroleum, Beijing 102249, China \\ ${ }^{4}$ Changqing Research Institute, China National Petroleum Corporation, Xi' an 710021, China \\ ${ }^{5}$ Changqing Institute of East Geophysical Company, China National Petroleum Corporation, \\ $X i$ an 710021, China
}

\begin{abstract}
We present an example of using converted-waves for characterizing onshore gas reservoirs in the Ordos basin in Northwest China. The Ordos basin is the largest gas province in China. The main gas reservoirs (about $3300 \mathrm{~m}$ in depth) are in upper Paleozoic sandstone that has low or reversed P-wave impedance and is immediately above a coal seam. This makes it very difficult to image the gas reservoirs using conventional P-wave data. Analysis of core, log and VSP data shows a weak PP reflection but a relatively strong PS-converted wave reflection, or both strong PP- and PS-reflections but with opposite polarity from the gas bearing sands, which indicates the potential of using PS-waves to image the gas reservoirs in the Ordos basin. Subsequently, thirteen seismic lines were acquired, processed and interpreted to verify the PP- and PS-responses, and two corresponding attributes (PP- and PS- amplitude ratio and polarity ratio) are used to map the reservoirs through joint PP and PS analysis.
\end{abstract}

Key words: gas reservoir; P- and PS-waves; well log; data processing

CLC number: P315.63 Document code: A

\section{Introduction}

The Zoeppritz equations for P-wave and PS reflected seismic waves are simplified by Aki and Richards (1980) assuming small elastic contrasts at the layer boundaries. The use of converted PS-waves to image reservoirs with low P-wave impedance but high S-wave impedance has attracted considerable interest in offshore exploration (e.g., MacLeod et al, 1999; Stewart et al, 2003). There are some cases of shear wave velocity inversion and stratigraphic inversion with PP and PS data (Mace et al, 2005; Wei et al, 2007). Roche et al (2005) provided an example of analysis for P-wave and con-

\footnotetext{
* Received 24 September 2008; accepted in revised form 2 February 2009; published 10 June 2009.

^ Corresponding author. e-mail: weixc@pepris.com
}

verted-wave seismic data. However, such applications to onshore basins have not been widely reported. We apply converted-waves to detecting gas reservoir in the Ordos basin in China. The Ordos basin is the largest gas province in China (Liu and Yang, 2000), and intensive exploration activities are going on there.

For many years, P-wave data have been used to image gas reservoirs in this area. However, there are many arguments against the use of P-wave data. A feasibility study using borehole data, including sandstone core samples and wireline logs, was carried out (Wei and Liu, 2003). The relationship between P- and S-wave velocities and porosity, gas saturation was established for the reservoir rock. Then some VSP data and surface three-component (3C) seismic data were used to demonstrate the characteristic P- and S-wave responses of actual gas-bearing sandstone. The results indicated that 
the joint PS-wave and P-wave responses can be used to detect gas reservoirs in the Ordos basin.

Thirteen lines of two-dimension (2D) threecomponent (3C) seismic data were acquired, processed and interpreted to evaluate the converted-wave technique. The key steps for data processing are static correction, noise attenuation and PS wave velocity analysis. For data interpretation, synthetic records were used to identify P- and PS reflections and related to the corresponding layer. Multicomponent wavefield attributes such as amplitude and velocity ratios are used to map the reservoirs through joint PP and PS analysis. The results are presented in this paper.

\section{Geological settings}

The Upper Paleozoic main reservoir systems in the Ordos basin were developed in continental sand bodies of the Shehezi group. The ancient landform is asymmetrical; material sources are relatively stable and abundant continental debris gathered in the lake and basin center continuously, resulting in widely distributed lake and river delta deposit systems in the middle of the basin. Because of the flat topography, the Lower Shehezi group sands are commonly found in the middle of the basin, and generally vary laterally. Owing to lateral accretion of meandering river sand bars, a near north-south complex sand band is developed, but because of channel migration, it shows great lateral variation. The reservoir lithology is mainly quartz and mineral debris sand. Its quartz content is on the low side, from $65 \%$ to $70 \%$ generally; the mineral debris is mainly coal sand or metamorphic rock and impurity content is high. The average porosity is $8 \%-10 \%$; permeability is only $0.2 \times 10^{-3}-1.0 \times 10^{-3}{\mu \mathrm{m}^{2}}^{2}$, and it is a typical low porosity and especially low permeability reservoir. Owing to a specific sediment source and diagenesis as well as depositional environment elements, a suite of thick sand reservoirs occur at the bottom of the Lower Shehezi group; cumulative sand thickness is $20 \mathrm{~m}$ or so generally, and can be up to $40 \mathrm{~m}$ to $50 \mathrm{~m}$. Reservoir lithology is mainly coarse quartz sand or pure quartz sand; quartz content is over $90 \%$ in general, and can be up to $95 \%$; pores are mainly inter-crystalline and inter-granular, but there are solution pores and tiny fissures. The quartz sand average

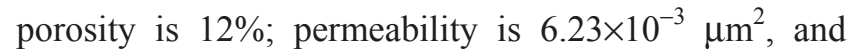
may be up to $561 \times 10^{-3} \mu \mathrm{m}^{2}$. It has medium porosity and medium-high permeability.

\section{Geophysical characteristics}

The geophysical properties of a sand reservoir, as well as the contrasts above and below the reservoir layer, form the basis of all geophysical exploration and estimation of reserves. The analysis of the reservoir's physical properties is a very important process in seismic inversion and correct identification of the geological meaning of seismic response.

The acoustic interval transit time in the sand reservoir layer is $230-260 \mu \mathrm{s} / \mathrm{m}$ in general; density is between $2.3 \mathrm{~g} / \mathrm{cm}^{3}$ and $2.5 \mathrm{~g} / \mathrm{cm}^{3}$; velocity is $3.8-4.4 \mathrm{~km} / \mathrm{s}$; average wave impedance is $0.88-1.1 \mathrm{~g} \cdot \mathrm{m}^{-2} \cdot \mathrm{s}^{-1}$. The ultrasonic transit time for mudstone (mud content over $80 \%$ ) is $220-240 \mu \mathrm{m} / \mathrm{s}$; density is $2.6-2.7 \mathrm{~g} / \mathrm{cm}^{3}$; velocity is $4.2-4.5 \mathrm{~km} / \mathrm{s}$; average wave impedance is 1.11-1.25 $\mathrm{g} \cdot \mathrm{m}^{-2} \cdot \mathrm{s}^{-1}$. Average velocity in siltstone is $4.55-4.70$ $\mathrm{km} / \mathrm{s}$; density is $2.6-2.65 \mathrm{~g} / \mathrm{cm}^{3}$; wave impedance is $1.18-1.25 \mathrm{~g} \cdot \mathrm{m}^{-2} \mathrm{~s}^{-1}$ (Tables $\left.1-3\right)$.

Table 1 Geophysical characteristics of various rock types in Shan 2 sequence, Yulin area

\begin{tabular}{lcccc}
\hline Type of rock & $\begin{array}{c}\text { Acoustic } \\
/ \mu \mathrm{s} \cdot \mathrm{m}^{-1}\end{array}$ & $\begin{array}{c}\text { Density } \\
/ \mathrm{g} \cdot \mathrm{cm}^{-3}\end{array}$ & $\begin{array}{c}\text { Velocity } \\
/ \mathrm{km} \cdot \mathrm{s}^{-1}\end{array}$ & $\begin{array}{c}\text { Impedance } \\
/ \mathrm{g} \cdot \mathrm{m}^{-2} \cdot \mathrm{s}^{-1}\end{array}$ \\
\hline Sandstone & 210 & 2.5 & 4.75 & $0.9-1.2$ \\
Mudstone & $220-260$ & 2.3 & $3.5-4.5$ & $0.8-1.04$ \\
Coal and mudstone & $400-450$ & $1.8-2.2$ & $2.2-2.8$ & $0.4-0.6$ \\
with coal & & & & \\
\hline
\end{tabular}

Table 2 Geophysical characteristics of various rock types in He 2 sequence, Uxin Qi area

\begin{tabular}{lcccc}
\hline Type of stone & $\begin{array}{c}\text { Acoustic } \\
/ \mu \mathrm{s} \cdot \mathrm{m}^{-1}\end{array}$ & $\begin{array}{c}\text { Density } \\
/ \mathrm{g} \cdot \mathrm{cm}^{-3}\end{array}$ & $\begin{array}{c}\text { Velocity } \\
/ \mathrm{km} \cdot \mathrm{s}^{-1}\end{array}$ & $\begin{array}{c}\text { Impedance } \\
/ \mathrm{g} \cdot \mathrm{m}^{-2} \cdot \mathrm{s}^{-1}\end{array}$ \\
\hline Sandstone & $215-225$ & $2.5-2.6$ & $4.4-4.65$ & $1.1-1.2$ \\
Muddy sandstone & $230-240$ & 2.5 & $4.2-4.5$ & $1.06-1.13$ \\
Mudstone & $250-265$ & 2.4 & $4.0-4.25$ & $0.96-1.02$ \\
\hline
\end{tabular}

Table 3 Geophysical characteristics of various rock types in $\mathrm{He}$ 2 sequence Sulige area

\begin{tabular}{lcccc}
\hline Type of stone & $\begin{array}{c}\text { Acoustic } \\
/ \mu \mathrm{s} \cdot \mathrm{m}^{-1}\end{array}$ & $\begin{array}{c}\text { Density } \\
/ \mathrm{g} \cdot \mathrm{cm}^{-3}\end{array}$ & $\begin{array}{c}\text { Velocity } \\
/ \mathrm{km} \cdot \mathrm{s}^{-1}\end{array}$ & $\begin{array}{c}\text { Impedance } \\
/ \mathrm{g} \cdot \mathrm{m}^{-2} \cdot \mathrm{s}^{-1}\end{array}$ \\
\hline Quartz sandstone & $230-260$ & $2.3-2.5$ & $3.8-4.4$ & $0.88-1.1$ \\
Muddy siltstone & $220-210$ & $2.6-2.65$ & $4.55-4.7$ & $1.18-1.25$ \\
Mudstone & $220-240$ & $2.6-2.7$ & $4.2-4.5$ & $1.1-1.25$ \\
\hline
\end{tabular}

The quantity of gas in sandstone has a very large influence on its P-wave impedance. Figure 1 shows that there can be a wide variation in the characteristics of the reflected P-wave. When sand porosity increases, the velocity and density decrease, and P-wave impedance decreases accordingly. For porosity and permeability properties in the area, when the porosity of sandstone rises from $10 \%$ to $14 \%$, P-wave velocity decreases from 
$4.0 \mathrm{~km} / \mathrm{s}$ to $4.4 \mathrm{~km} / \mathrm{s}$ generally, and density changes from $2.35 \mathrm{~g} / \mathrm{cm}^{3}$ to $2.45 \mathrm{~g} / \mathrm{cm}^{3}$. For the gas layer, the P-wave velocity of high permeable sandstones may decrease to $3.8 \mathrm{~km} / \mathrm{s}$. Therefore sandstone cannot be distinguished from pure mudstone. However, for sandy mudstone or muddy sandstone with mud content $20 \%$ to $80 \%$, the P-wave velocity is usually $4.3-4.6 \mathrm{~km} / \mathrm{s}$, and density is $2.5-2.6 \mathrm{~g} / \mathrm{cm}^{3}$. Thus, the rapid decrease of P-wave impedance provides a method for identifying high porosity-permeability gas reservoirs in P-wave impedance profiles.
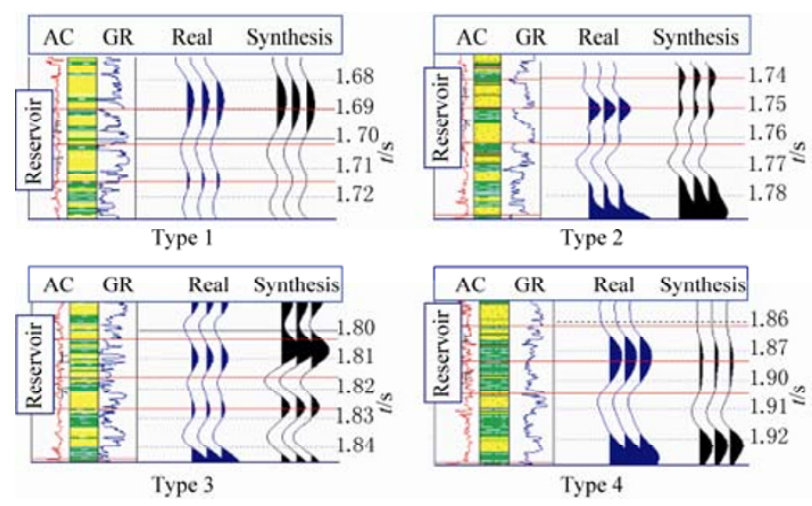

Figure 1 PP-wave Reflection waveforms from the gas reservoir.

When identifying a gas reservoir from well-log data, a low impedance layer correlated with existing wells is interpreted as the gas layer. But a low velocity strip separated by high impedance should be treated carefully, because it is highly possible that this is caused by increased errors. A P-wave impedance decrease due to gas is similar to that due to high permeability of water bearing, so it is possible to mistake one for the other without additional information. Since S-wave impedance is hardly influenced by the presence of gas, joint PP and PS studies have great significance in this area. For this purpose, a feasibility study of full-wave modeling based on core and well-logs has been carried out to evaluate the potential of using converted-waves for characterizing gas sands.

\section{Feasibility study}

\subsection{Core analysis}

Forty-five core samples (Figure 2) were tested in the laboratory, as measured by Institute of Geology and Geophysics, Chinese Academy of Sciences, for Changqing Research Institute, China National Petroleum Corporation. The cores were filled with four degrees of gas saturation: $100 \%, 65 \%$ to $75 \%, 35 \%$ and $0 \%(100 \%$ water saturation). Both $\mathrm{P}$ - and $\mathrm{S}$-wave velocities were measured at efficient pressure (confining pressure minus pore pressure) between $35 \mathrm{MPa}$ and $42 \mathrm{MPa}$, and temperatures between $78^{\circ} \mathrm{C}$ and $100^{\circ} \mathrm{C}$. Experimental frequencies were $800 \mathrm{kHz}$ and $600 \mathrm{kHz}$ for P- and S-waves, respectively. Testing was carried out as follows: (1) eight core samples numbered 1 through 8 were tested under $90{ }^{\circ} \mathrm{C}$ temperature and $42 \mathrm{MPa}$ efficient pressure; (2) eighteen core samples numbered 9-20 and 25-30 were tested under $95{ }^{\circ} \mathrm{C}$ temperature and $45 \mathrm{MPa}$ efficient pressure; (3) seven core samples numbered 21-24 and 41-43 were tested under $100{ }^{\circ} \mathrm{C}$ temperature and 47 $\mathrm{MPa}$ efficient pressure; (4) twelve core samples numbered $31-40,44$ and 45 were tested under $78^{\circ} \mathrm{C}$ and 30 $\mathrm{MPa}$ efficient pressure.

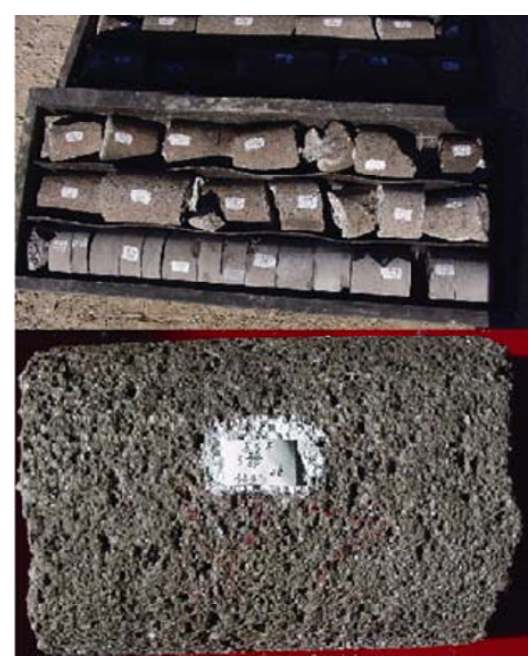

Figure 2 Typical core samples for velocity measurements.

The variation of measured $\mathrm{P}$ - and S-wave velocities with porosity is shown in Figure 3 for all four different saturations. Even if there is lot of scatter in the data from different samples, the variation trends in seismic wave velocity is consistent with Gassmann predictions for gas saturation. The P-wave velocity clearly decreases as the porosity increases, and also decreases as the gas saturation increases. However, there is less variation in the S-wave velocity than that in P-wave velocity. Otherwise, it shows some small random variations in S-wave velocity as saturation changes.

\subsection{Well log data analysis}

We have also examined the relationship of the Pand S-wave velocities with porosity and saturation by using log data. Wireline logs from eleven boreholes are examined to identify the log characteristics of the gas 
reservoirs. Figure 4 shows the cross plot of the P- and S-wave sonic velocities against the logged porosity. Again it shows a clear decrease of the P-wave velocity and S-wave velocity as porosity increases. However, there is less variation in the S-wave velocity than that in P-wave velocity. This confirms the findings of core data analysis. Figure 5 shows the variation of P-wave sonic velocity for gas and water saturations. The P-wave velocity saturated with gas decreases more rapidly than that saturated with water.
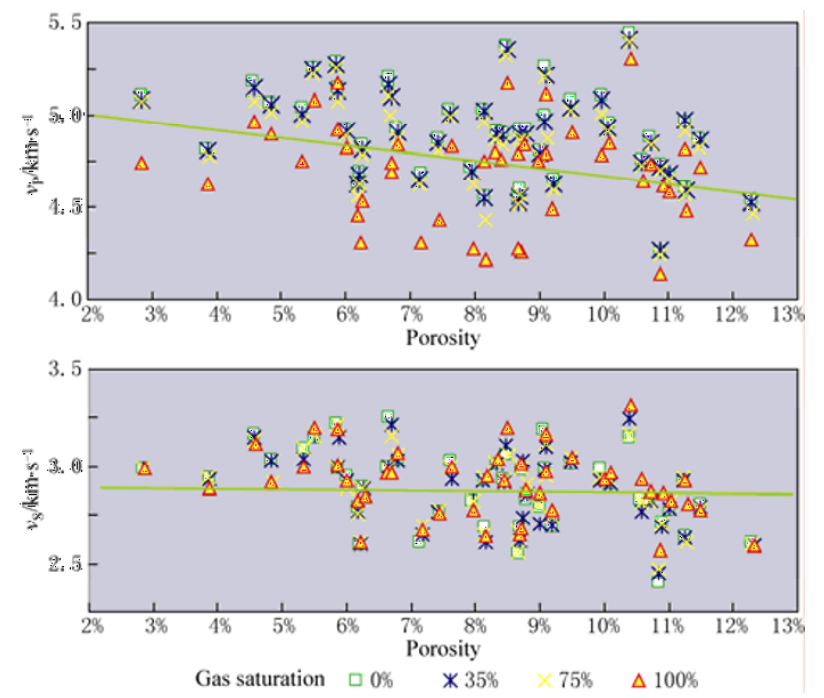

Figure 3 Variations of P- and S-wave velocities with porosity and gas saturation from core measurements.

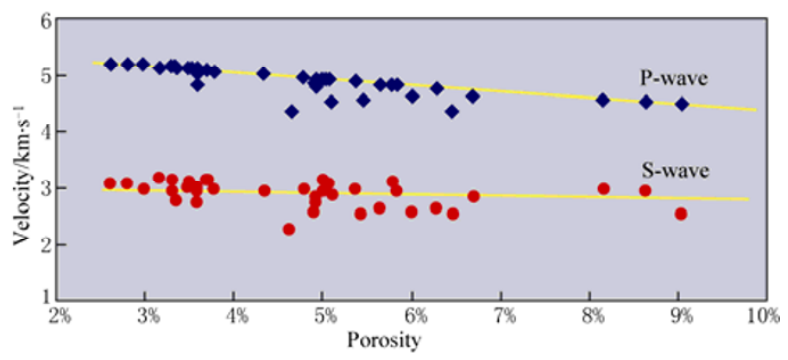

Figure 4 Variations of P- and S-wave velocities with porosity from wireline logs.

\section{Application to field data}

\subsection{Data acquisition}

The P-wave reflected energy from the coal seam can be observed in the vertical component and the corresponding PS conversion can be observed in the horizontal component. There are several kinds of noise on the records. For example, ground rolls and refracted waves are obviously present. Because the near-surface in the area is characterized by low velocity loose soil,
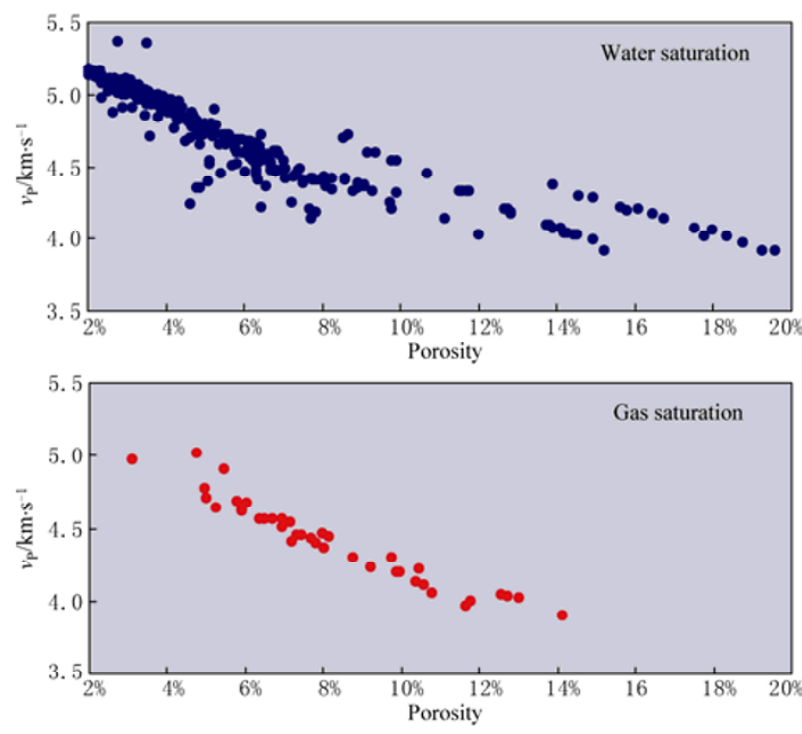

Figure 5 Variation of P-wave velocity with gas and water saturations.

this causes the presence of a significant amount of ground rolls and refracted waves, as well as complex statics in the data. These are key issues that affect the quality of the stacked sections. However, on the other hand, the presence of the low-velocity near-surface also gives rise to a natural separation of PP- and PS-waves in the vertical and horizontal components, which simplifies wave-field separation. The reason is that the rays of reflection wave are nearly vertical in low-velocity layer near surface from deep layers, so all PP-wave energy is almost in vertical component and PS-wave energy in horizontal components. Despite the strong coherent noise, converted-waves can be observed from intermediate to far offsets even in raw shot records of the horizontal component. Note that there are no convertedwaves in the near-offsets, as expected.

\subsection{Data processing}

For PS-converted wave data, there are three key steps in the processing flow: static correction, noise reduction and non-hyperbolic velocity analysis.

Static correction is a major challenge for seismic exploration in mountains and desert terrain with loose rocks or soil. For PS-converted waves, it is even more important in data processing. A special method is developed for static correction in loose terrain, which utilizes a continuous velocity model (Wei and Liu, 2002).

PS-wave statics is a big issue for land PS data. In this area, the second arrival energy presents on horizontal component data (Figure 6). We think this is PS-converted refraction wave. The waves travel down-going and slid- 
ing along refracted layer with P-wave velocity but up-going with S-wave velocity. So this wave can be used to calculate S-wave velocity during P-S wave receiver statics. The first step is to pick the PS-refracted arrivals, and then estimate the S-wave velocity from the PS-arrival at the near surface. The last step is to calculate the S-wave static values for each receiver. Figure 6 shows the improved shot records of the horizontal component after applying PS-wave static correction.
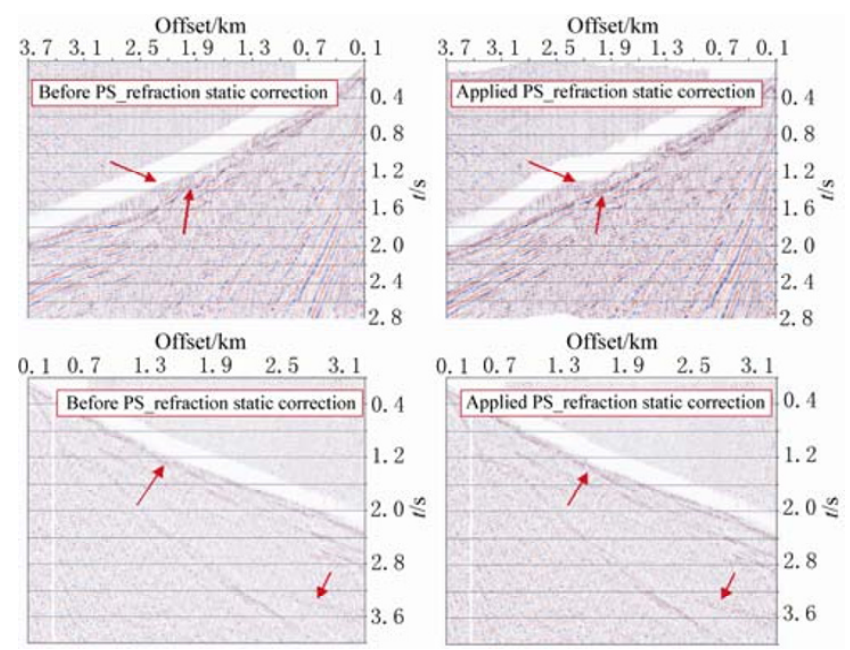

Figure 6 Effects of applying PS-wave static corrections for the horizontal component.

There are several types of noises in the PS records. Conventional velocity filters are used to suppress the coherent noise, and the success of this step mainly depends on the prior velocity functions. In this case, the VSP data are used to extract the prior velocity functions for filtering purposes.

Non-hyperbolic moveout analysis is required to handle the converted-waves in the intermediate to far offsets. The three-term non-hyperbolic moveout equation (Li and Yuan, 2001) is used to fulfill this purpose. Time-variation CCP (common converted point) gather is used in PS velocity analysis and PS mapping. One example of semblance with super CCP gather is shown in Figure 7.

Using these steps described above, the quality of the stacked sections is quite good (Figure 8). Comparison of the PS-wave stacked section with the PP-wave stacked section shows that reflected events appear more clearly, particularly between $1000 \mathrm{~ms}$ to $2000 \mathrm{~ms}$.

\subsection{Data analysis and results}

PP and PS event registration is very important in PP and PS joint interpretation. At first, we chose two

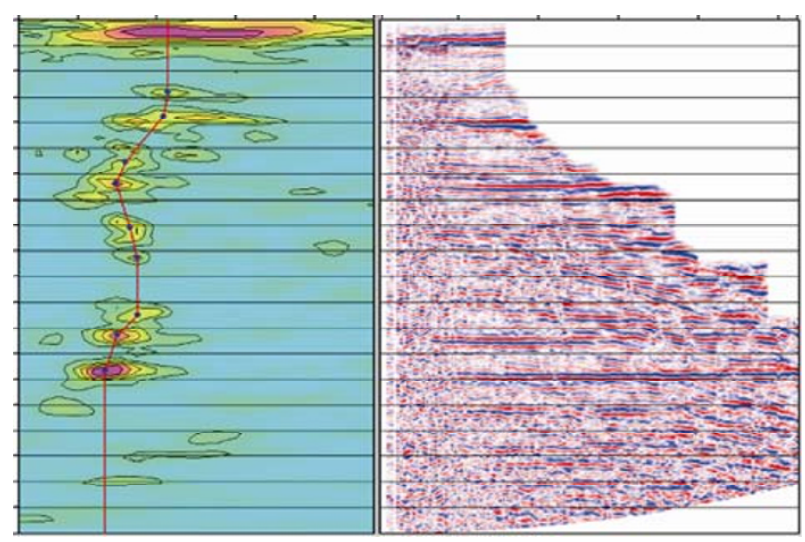

Figure 7 Converted-wave velocity analysis. Left panel is velocity spectrum and right panel is moveout corrected gather.

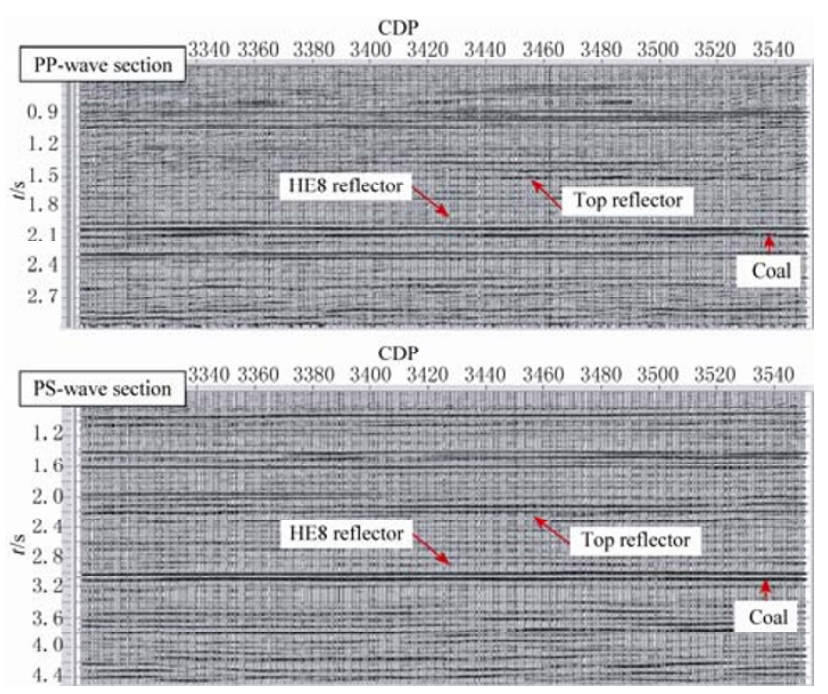

Figure 8 PP- and PS-wave stack sections.

strong events (Figure 8) as standard layers, where the main objects are situated. The coal-seam reflection immediately below the target (HE8) is very strong, and presents over the whole area in both PP- and PS-sections (Figure 8). It acts as a good reference horizon and simplifies the analysis procedure, such as event registration and amplitude normalization as well as layer tracking. Reservoir HE8 is immediately above the coal seam, making horizon tracking easier. Then according to logging and VSP data, P- and S-wave interval velocities in depth domain are determined and used in synthetic records. The interval velocities are adjusted iteratively to make synthetic records best matching with real PP- and PS data. The optimized P- and S-wave velocities are used in depth conversion.

After the coal-seam event is registered and the amplitude is normalized for both the PP- and PS-sections, 
two attributes are calculated for identifying gas sands along the target horizon. The attributes are relative amplitude ratio $\left(a_{\mathrm{PS}}-a_{\mathrm{P}}\right) / a_{\mathrm{PS}}$ and relative polarity. Note that the PP and PS-reflections have the same polarity as the background matrix of sand and mudstone. The velocity ratio can also be used to deduce the fluid in a reservoir. Figures 9 and 10 show the correlation of P- and S-wave velocities for different lithology and inversion results of velocity ratio from real data respectively.

The combinations of these attributes are good indicators of six types of rock and their pore content (Table 4). The first type is high quality gas sands characterized by strong PP and PS amplitudes (PP stronger than PS) with opposite polarity, where the presence of gas is so significant that the velocity of the gas bearing sands is lower than the overlying mudstone (a polarity reversal). In this area, the porosity should be $14 \%$ for this case.

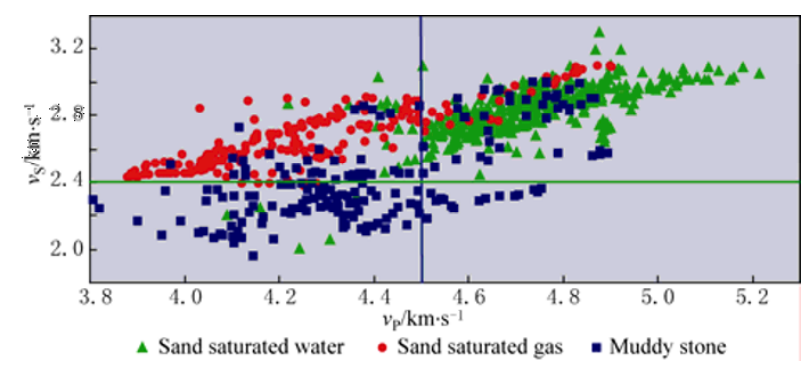

Figure 9 Correlation of $\mathrm{P}$ - and S-wave velocities for different lithologies.

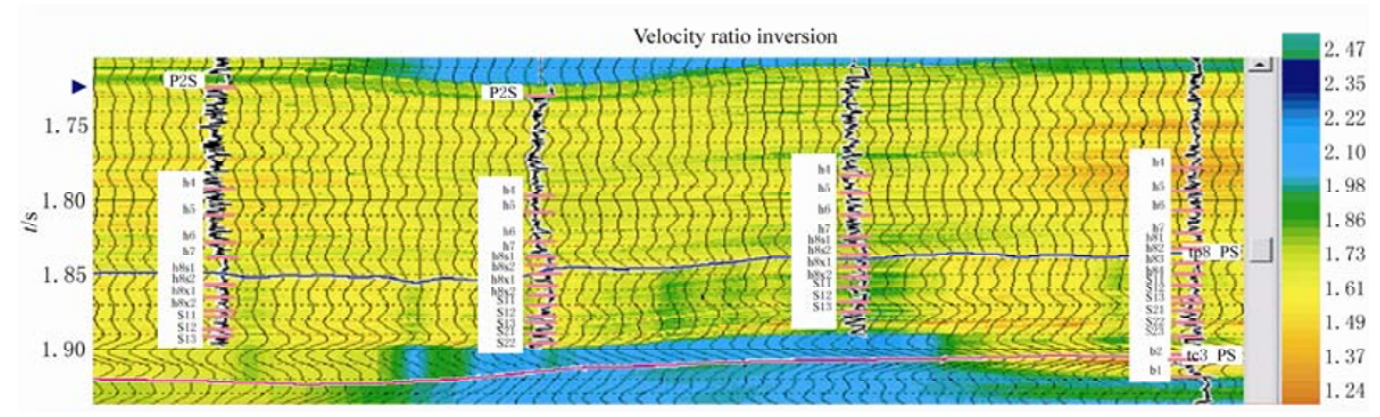

Figure 10 Inverted P- and S-wave velocity ratios from PP- and PS stacked sections.

Table 4 PP- and PS indicators for gas sands

\begin{tabular}{|c|c|c|c|c|c|c|}
\hline Types & PP-wave & PS-wave & Polarity of PP and PS & $v_{\mathrm{P}} / v_{\mathrm{S}}$ & Frequency & Contents \\
\hline 1 & Strong & Strong & Opposite & Low & Low & Gas (best) \\
\hline 2 & Weak & Strong & Opposite or same & Low & Low & Gas (good) \\
\hline 3 & Intermediate & Weak & Opposite or same & Low & Low & Gas (good) \\
\hline 4 & Intermediate & Strong & Same & Intermediate & Intermediate & Gas (less) or water \\
\hline 5 & Strong & Strong & Same & high & High & Dry \\
\hline 6 & Weak & Weak & Opposite or same & high & High & No sand \\
\hline
\end{tabular}

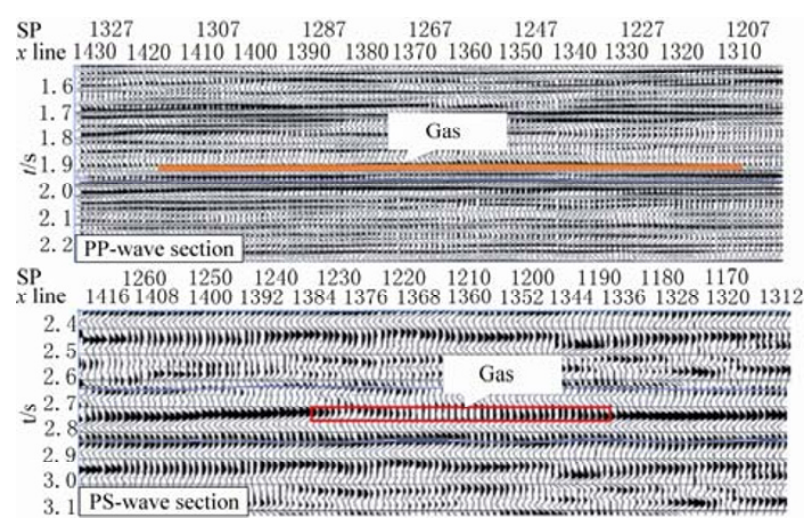

Figure 11 Seismic responses showing a strong P-wave and weak PS-wave response indicates sandstone grit surrounded by siltstone, the third type of gas reservoir in Table 4.
The second type is medium-quality gas sands characterized by weak PP amplitude but strong PS amplitude with the same polarity. The velocity of P-wave is nearly the same in gas-bearing sands and mudstone. The porosity of gas-bearing sands would be about $11 \%$ in the studied area.

The third type is special gas sand, sandstone grit saturated with gas covered by siltstone. It is characterized by strong PP and weak PS amplitudes. Figure 11 shows the corresponding seismic characteristics.

The fourth type is poor quality gas-bearing sands characterized by strong PS amplitude and intermediate PP amplitude with the same polarity for PP and PS-waves. The porosity is about $8 \%$ or lower. Sometimes this may indicate water-bearing sands. 
The fifth type is dry sand (no gas), characterized by strong PP and PS amplitudes with the same polarity, reflecting the background matrix, where no gas is present to alter the velocity of the sand.

If both of PP-and PS-wave amplitudes are weak, normally this indicates no sands present (sixth type - all mudstones). Therefore, a basic strategy for mapping gas sands can be formulated as follows: Firstly, search for strong amplitude reflections from both PP and PS-sections but with opposite polarity. A weak PS-wave amplitude but strong PP-wave amplitude may also indicate gas reservoirs.

\section{Discussion and conclusions}

We have performed an integrated study of the seismic response of the gas reservoirs in the Ordos basin using core, log and seismic data. The feasibility study shows that it is difficult to predict gas-bearing sandstone using P-wave only, but it is possible using both $\mathrm{P}$-wave and PS-wave responses. High quality P- and converted PS-wave sections are a precondition. There are three key steps in processing PS-converted data in the area, that is, near surface static correction, noise attenuation and non-hyperbolic velocity analysis. Analysis of the core and logging data shows that the variation in porosity and gas saturation has a strong effect on the P-wave velocity of the reservoir sandstone (the sandstone gas reservoirs are surrounded by mudstone or siltstone), whereas the effect on the S-wave velocity is small.

The core and log analysis as well as field data indicates that it may be possible to identify six types of sandstones in the studied areas by joint PP and PS analysis: (1) a strong PP- and PS-wave response but with opposite polarity indicates a high-quality gas sand; (2) a weak PP-wave but strong PS-wave response with the same polarity indicates a fairly good gas-bearing sand; (3) a weak PS-wave energy but strong PP-wave energy indicates a special type of gas reservoir consisting of sandstone grit surrounded by siltstone; (4) an intermediate PP-wave and strong PS-wave response with the same polarity indicates a poor gas-bearing sandstone or water-saturated sandstone; (5) strong PP- and PS-waves responses with the same polarity indicates dry sand with no gas; (6) both weak PP- and PS-waves responses indicate mudstones containing little sand.

\section{References}

Aki K and Richards P G (1980). Quantitative Seismology: Theory and Methods. W H Freeman and Company, Cambridge Press, Cambridge, United Kingdom, 144-154.

Liu S and Yang S (2000). Upper Triassic-Jurassic sequence stratigraphy and its structural controls in the western Ordos basin, China. Basin Research 12: $1-18$.

Li X-Y and Yuan J (2001). Accuracy and sensitivity analysis for estimating anisotropic parameters from 4C seismic. 71st SEG Meeting. San Antonio, USA, Expanded Abstracts, 869-872.

Mace D, Agullo Y, Labat K, Tonellot T, Bourgeois A and Lavielle M (2005). Joint PP and PS stratigraphic inversion for prestack time migrated multicomponent data. EAGE/SEG Research Workshop "Multicomponent Seismic-Past, Present and Future". European Association of Geoscientists and Engineers, Pau, France, P16.

MacLeod M, Hanson R, Hadley M, Reynolds K, Lumley D, McHugo S and Probert T (1999). The Alba field OBC seismic survey. 69th SEG Meeting. Houston, USA, Expanded Abstracts, 725-727.

Roche S, Wagaman M and Watt H (2005). Analysis of P-wave and converted-wave seismic data, Anadarko basin, Oklahoma, USA. EAGE/SEG Research Workshop "Multicomponent Seismic-Past, Present and Future". European Association of Geoscientists and Engineers, Pau, France, A19.

Stewart R R, Gaiser J E, Brown R J and Lawton D C (2003). Converted-wave seismic exploration: Applications. Geophysics 68(1): 40-57.

Wei X and Liu Y (2002). The study of static correction in desert and loose terrain. 72nd SEG Meeting. Salt Lake Cities, USA, Expanded Abstracts, 2.249-2 252 .

Wei X and Liu Y (2003). Case study of using converted PS-waves to image gas reservoirs in Ordos basin, China. 73rd SEG Meeting. Dallas, USA, Expanded Abstracts, 769-772.

Wei X C, Wang J M and Chen T S (2007). Inversion of shear wave interval velocity on prestack converted wave data. Acta Seismologica Sinica 20(2): 180-187. 